\title{
Characterization of cytomegalovirus pp65-HLA-A24 peptide- specific CTL lines from metastatic melanoma patients
}

\author{
YASUTO AKIYAMA $^{1}$, SACHIKO TAI ${ }^{1}$, MASARU KOMIYAMA ${ }^{1}$, MASAKO TAKIKAWA ${ }^{1}$, CHIE OHSHITA $^{1}$, \\ AKIFUMI YAMAMOTO $^{4}$, NAOYA YAMAZAKI ${ }^{3}$ and YOSHIO KIYOHARA ${ }^{2}$ \\ ${ }^{1}$ Immunotherapy Division, Shizuoka Cancer Center Research Institute; ${ }^{2}$ Department of Dermatology, Shizuoka \\ Cancer Center Hospital, 1007 Shimonagakubo, Nagaizumi-cho, Sunto-gun, Shizuoka 411-8777; ${ }^{3}$ Department \\ of Dermatology, National Cancer Center Hospital, 5-1-1 Tsukiji, Chuo-ku, Tokyo 104; ${ }^{4}$ Department of \\ Dermatology, Saitama Medical University, 38 Moroyama, Iruma-gun, Saitama 350-0495, Japan
}

Received December 8, 2008; Accepted March 3, 2009

DOI: $10.3892 /$ or_00000423

\begin{abstract}
Because of advances in immunological technology for detecting a very small number of blood CTL cells, clinicians have been able to monitor cellular immunity against CMV and evaluate the status of CMV infections in highly advanced cancer patients or transplant recipients. Our previous study using healthy volunteer PBLs revealed a significant increase in CMV HLA-A24 tetramer ${ }^{+}$CTLs after stimulation in vitro with autologous DCs. However, the efficiency of CMV A24 peptide-specific CTL expansion in highly advanced cancer patients has yet to be studied in detail. In the present study, we tried to characterize and expand HLA-A*2402 CMVpp65 peptide (QYDPVAALF)-specific tetramer ${ }^{+}$ CTLs from HLA-A* $2402^{+}$metastatic melanoma patients, and eventually demonstrated that expansion efficiency was closely related to both post-stimulation CMV tetramer frequency and anti-CMV IgG titer. This is a novel finding regarding in vitro CMVpp65-A24 peptide-specific CTL expansion based on metastatic cancer patient-derived PBLs. Interestingly, the current results using metastatic melanoma PBLs showed a much higher frequency of CMVpp65-A24 tetramer ${ }^{+}$CTLs and expansion efficiency than in healthy volunteers. Finally, we were successful in cloning CMVpp65 HLA-A24 peptidespecific TCR cDNAs from in vitro expanded CTL lines derived from melanoma patients. Additionally, CMVpp65 HLA-A24 peptide-specific TCR cDNA was transduced into naive $\mathrm{T}$ cells from patients and functionally reconstructed. The results showed that cloned CMV-specific TCR genes were efficient in reconstituting specific anti-CMV activity
\end{abstract}

Correspondence to: Dr Yasuto Akiyama, Immunotherapy Division, Shizuoka Cancer Center Research Institute, 1007 Shimonagakubo, Nagaizumi-cho, Sunto-gun, Shizuoka 411-8777, Japan

E-mail: y.akiyama@scchr.jp

Key words: CMVpp65 HLA-A24 epitope, CMV-specific CTL line, anti-TCR MoAb sorting, TCR gene cloning, electroporation and might be good tools for adoptive immunotherapy against CMV infections.

\section{Introduction}

The development or re-activation of cytomegalovirus (CMV) infections in immunocompromised recipients of transplantations and advanced staged cancer patients who received a high dose of chemotherapy or irradiation remains difficult to cure and is still a critical issue in the clinical oncology field. Although the ganciclovir, anti-CMV drug is considered effective against CMV, it can not totally eradicate all viral particles from infected cells and has significant adverse effects when taken long-term. Meanwhile, an immunological therapeutic approach, the reconstitution of cellular immunity by the adoptive transfer of ex vivo expanded cytotoxic $\mathrm{T}$ lymphocytes (CTLs), is expected to eradicate and cure CMV infections in immunocompromised patients (1-3).

In our previous studies, CMVpp65 matrix protein (aa 341-349) peptide-specific CTL lines were obtained from peripheral blood lymphocytes (PBLs) of 12 human leukocyte antigen (HLA)-A24+ healthy donors and the frequency of HLA-A*2402-CMVpp65 peptide tetramer ${ }^{+}$CTLs was found to be strongly correlated to the amount of IFN $-\gamma$ produced by those CTL lines (4). In the present study, in order to evaluate the efficacy of adoptive CTL therapy in highly advanced (metastatic) cancer patients, we performed an in vitro expansion of CMVpp65-A24 peptide-specific CTL lines from metastatic melanoma patients, and investigated the correlation of expansion efficiency with immunological parameters such as the frequency of tetramer ${ }^{+}$CTLs staining and the anti-CMV IgG titer.

Additionally, we were successful in cloning specific T-cell receptor (TCR) cDNA of a CMVpp65 HLA-A24 peptidespecific CTL line and characterized its cytotoxic function.

\section{Materials and methods}

Reagents and cell lines. Recombinant human (rh) granulocyte macrophage colony-stimulating factor (GM-CSF), rhinterleukin (IL)-2, rhIL-4, rhIL-7 and tumor necrosis factor (TNF)- $\alpha$ were purchased from Pepro Tech, Inc. (Rocky Hill, 
NJ, USA). FITC and PE-labeled mouse anti-human IFN- $\gamma$ antibodies were purchased from Pharmingen (San Diego, CA). HLA*2402 CMVpp65 (QYDPVAALF) and human immunodeficiency virus (HIV) (RYLRDQQLL) tetramers were supplied from MBL (Nagoya, Japan). TCR Vß repertoire kit and FITC-labeled anti-specific TCRVß repertoire monoclonal antibodies (MoAbs) were purchased from Beckman Coulter, Inc. (CA, USA). TISI cells, HLA-A24+ Epstein-Barr (EB) virus-transformed B cell line and T2-A24 cells, the TAP (transporter associated with antigen processing)deficient $\mathrm{B}$ and $\mathrm{T}$ hybrid cell line $\mathrm{T} 2$ transfected with the HLA-A*2402 gene, were kindly supplied by TakaraBio Co., Ltd. and Dr Kuzushima, Aichi Cancer Center Research Institute, respectively.

Synthetic peptides. The melanoma-associated HLA-A24restricted synthetic peptides used in the present study were CMVpp65 matrix protein 341-349 (QYDPVAALF), CEA 652-660 (TYACFVSNL) and HIV 584-592 (RYLRDQQLL).

Measuring anti-CMV IgG Ab titer in plasma. Anti-CMV IgG $\mathrm{Ab}$ titers in samples of plasma from melanoma patients were measured using anti-CMV IgG-specific EIA kit (Denka Seiken Co., Tokyo, Japan). CMV whole virions inactivated by UV light from strain AD-169 were used as coated viral antigens in the present kit. An anti-CMV IgG index was calculated as the maximal dilution value divided by 100 in plasma samples. The cut-off value was 2.0.

Generation of dendritic cells (DCs) from blood monocytes. All peripheral blood mononuclear cells (PBMCs) were derived from HLA-A $* 2402^{+}$metastatic melanoma patients (The clinical research using PBMCs from melanoma patients was approved by the Institutional Review Board of National Cancer Center, Tokyo and Shizuoka Cancer Center, Shizuoka, Japan. All patients gave written informed consent). PBMCs were cultured at $2.5-5 \times 10^{6}$ cells $/ \mathrm{ml}$ in RPMI-1640 medium supplemented with $2 \mathrm{mM}$ L-glutamine, $100 \mathrm{U} / \mathrm{ml}$ penicillin and $100 \mu \mathrm{g} / \mathrm{ml}$ streptomycin, $50 \mu \mathrm{g} / \mathrm{ml}$ gentamycin, $50 \mu \mathrm{M} ß$-mercaptoethanol, $1 \mathrm{mM}$ sodium pyruvate and $5 \% \mathrm{AB}$ human serum (Lonza, Walkersville, MD) (referred to as DC medium) for $90 \mathrm{~min}$ at $37^{\circ} \mathrm{C}$. After incubation, non-adherent cells were collected and stored at $-80^{\circ} \mathrm{C}$ until used. The adherent monocyte-enriched population was cultured in the presence of $40 \mathrm{ng} / \mathrm{ml}$ of rhGM-CSF and rhIL-4. TNF- $\alpha$ was added to the culture on day 5 at a final concentration of $10 \mathrm{ng} / \mathrm{ml}$. After 7 days of culture, cells were harvested and used for CTL induction cultures.

CTL induction cultures (stimulation with DCs and/or T2-A24 cells). Harvested DCs were incubated with $50 \mu \mathrm{g} / \mathrm{ml}$ of CMV pp65 A24 peptide and $3 \mu \mathrm{g} / \mathrm{ml}$ of B2-microglobulin (Sigma, St. Louis, MO) in PBS with calcium and magnesium containing $1 \%$ human serum albumin for $2 \mathrm{~h}$ at $37^{\circ} \mathrm{C}$. Thirty Gy-irradiated peptide-pulsed DCs were cultured with nonadherent cells derived from PBMCs at a ratio of 1:10-1:100 in DC medium with $10 \mathrm{ng} / \mathrm{ml}$ of rhIL-7. On days 2 and 5, rhIL-2 was added to the culture at a final concentration of $3 \mathrm{ng} / \mathrm{ml}$. After 7 days of culture, CTLs were harvested and restimulated with CMV peptide-pulsed DCs for another 7 days.
Additionally, cultured CTL cells were boosted with 2 rounds of stimulation with CMVpp65 A24 peptide-pulsed T2-A24 cells weekly at a ratio of 1:10. Finally, expanded peptidespecific CTLs were utilized for various experiments including tetramer staining and cell sorting.

Tetramer staining. Cultured CTLs were stained with both FITC-anti-CD8 MoAb and PE-labeled HLA-A24 CMVpp65 or HIV tetramer as previously described. Cells were analyzed with a flow cytometer (FACS Calibur, BD Science, CA).

$T C R$ repertoire staining by anti-TCR MoAb. The staining profile of CTLs during the expansion procedure was monitored using a TCR Vß repertoire kit, and after DC plus T2-A24 cell-based expansion, major populations positively stained with the specific anti-TCR antibody were determined.

CTL killing assay. The CTL killing assay was performed according to the DELFIA non-radioactive cytotoxicity assay (Perkin-Elmer, Inc., Waltham, MA). Briefly, target cells (peptide-pulsed TISI cells) were labeled with a fluorescence enhancing ligand, BATDA, and mixed with effector cells at an E/T ratio of 10 for $4 \mathrm{~h}$. The supernatant collected from each CTL culture was incubated with europium solution, and the EuTDA level was measured in real-time with a fluorometer, ARVOsx-2 (Perkin-Elmer, Inc.).

Intracellular IFN- $\gamma$ staining. In some melanoma cases after cultured CTLs were pre-incubated with CMVpp65 peptidepulsed or non-pulsed TISI cells for $4 \mathrm{~h}$, stimulated CTLs were stained intracellularly with anti-human IFN- $\gamma$ MoAb, HLA-A24 CMVpp65 peptide-specific tetramer and/or antispecific TCR MoAb. Stained cells were analyzed on a flow cytometer.

CTL sorting by MACS. TCR MoAb-based CTL sorting was performed using the autoMACS (magnetic cell sorting) system (Miltenyi, Germany). Briefly, we used FITC-labeled antiTCRBV4 or TCRBV19 MoAb as the primary antibody, and anti-FITC MoAb microbeads as the secondary antibody. The purity of specific $\mathrm{TCR}^{+}$CTLs was $>95 \%$ (data not shown). Purified CTLs were sequentially used for PCR cloning of TCR cDNA.

TISI-stimulated IFN- $\gamma$ production from peptide-specific CTL line sorted by TCR-specific MoAb. The expanded CTL line from case MEL-2 was sorted by FITC-labeled anti-TCRBV4 $\mathrm{MoAb}$ using the autoMACS system. After an over-night incubation with DC medium, sorted CTLs $\left(1 \times 10^{5}\right)$ and peptidepulsed TISI cells $\left(1 \times 10^{5}\right)$ were co-incubated in a roundbottomed 96-well microculture plate for $24 \mathrm{~h}$. Finally, supernatants were collected and IFN- $\gamma$ levels were measured using an ELISA kit specific for human IFN- $\gamma$ (Biosource, Camallilo, CA).

PCR cloning and sequencing of CMVpp65 peptide-specific TCRBV gene. Total RNA of sorted CTLs was prepared with a kit, Nucleospin RNA II (Macherey-Nagel, Germany), and aliquots of $2 \mu \mathrm{g}$ were subjected to reverse transcription using an oligo(dT) primer and SuperScript II (Invitrogen, CA). The 
first strand cDNA was amplified by PCR using KOD polymerase (Toyobo, Japan) according to the manufacturer's instructions and coding region-specific primers for MEL-2; TCRBV4 and TCRBC1, MEL-3; TCRBV19 and TCRBC1, and MEL-4; TCRBV4 and TCRBC2, respectively. The primer sequences are as follows: 5'-GCTAGCATGGGCTGC AGGCTGCTCTGC-3' for TCRBV4, 5'-TGCACTATGAGC AACCAGGTGCTCTGC-3' for TCRBV19, 5'-TCAGAAAT CCTTTCTCTTGACCATGGC-3' for TCRBC1, and 5'-CTA GCCTCTGGAATCCTTTCTCTTGAC-3' for TCRBC2. The PCR product was separated on a $1.5 \%$ agarose gel, and the band of appropriate size (bp) was excised and extracted from the gel. The recovered DNA fragment was cloned into the plasmid pCR-Blunt (Invitrogen, CA), and its DNA sequence was determined using BigDye Terminator reagent and a 3130xl Genetic Analyzer (Applied Biosystems, CA). To confirm DNA sequences, $>12$ independent clones were analyzed for each TCR gene. The confirmed cDNA sequences for each TCR gene were analyzed by a WEB tool of IMGT (JunctionAnalysis, http://imgt.cines.fr/).

Construction of expression plasmids. The CMVpp65 A24 peptide-specific TCR gene from case MEL-2 cloned in pCR-Blunt was digested with PstI and blunted with T4 DNA polymerase (Takara, Japan), and then digested with BamHI. The resultant DNA fragment was cloned into the blunted NheI-BglII site of the pmax expression plasmid (Amaxa, Germany).

TCR gene transduction into primary naive T cells. A plasmid vector named pmax was utilized for making constructs containing GFP or cloned specific TCR genes. The T cell transfection kit (Nucleofector ${ }^{\mathrm{TM}}$, Amaxa, Cologne, Germany) and Nucleofector device (Amaxa) were used according to the manufacturer's instructions. Prior to electroporation, all lymphocytes including $\mathrm{T}$ cells derived from case MEL-2 were stimulated with anti-CD3 $(2 \mu \mathrm{g} / \mathrm{ml})$ and CD28 MoAb $(1 \mu \mathrm{g} / \mathrm{ml})$ for 5 days and collected for a gene transduction. The expression of TCR protein was analyzed with a flow cytometer using FITC anti-TCRBV4 MoAb.

IFN- $\gamma$ production by specific TCR gene-transduced naive $T$ cells. Two days after electroporation, naive $\mathrm{T}$ cells transduced with GFP or a specific TCR gene were harvested and incubated with CMVpp65 or other peptide-pulsed TISI cells for $24 \mathrm{~h}$. The supernatant was collected and the IFN- $\gamma$ level was measured using an ELISA kit specific for human IFN- $\gamma$ (Biosource).

\section{Results}

Tetramer $^{+}$CTL induction and expansion. After the expansion of CMVpp65 A24 peptide-specific CTLs, the frequency of CMVpp65 tetramer ${ }^{+}$CTLs increased to $18.6 \%$ (mean of 12 cases) compared with before stimulation $(<0.1 \%)$ (Table I). The highest frequency of tetramer ${ }^{+}$CTLs was seen in the MEL-2 case just after DC stimulation 2 times which also showed the most potent CTL expansion (data not shown). The total CMVpp65 tetramer ${ }^{+}$CTL counts exhibited efficient expansion ranging from 14.8 to 988.4 (mean, 356.5) fold. Additionally, we investigated the relevance of expansion
Table I. CMV-based immunological parameters in melanoma patients.

\begin{tabular}{|c|c|c|c|c|}
\hline \multirow[b]{2}{*}{ Cases } & \multicolumn{2}{|c|}{$\begin{array}{l}\text { Tetramer } \\
\text { ratio }(\%)\end{array}$} & \multirow{2}{*}{$\begin{array}{l}\text { Expansion } \\
\text { of tetramer } \\
\text { CTL\# (fold) }\end{array}$} & \multirow{2}{*}{$\begin{array}{c}\text { Anti-CMV } \\
\text { IgG Ab }\end{array}$} \\
\hline & Pre & Post & & \\
\hline MEL-1 & 0.04 & 0.7 & 14.8 & $<2.0$ \\
\hline MEL-2 & 0.15 & 65.9 & 988.4 & 63.9 \\
\hline MEL-3 & 0.06 & 11.2 & 326.7 & 29.1 \\
\hline MEL-4 & 0.11 & 35.0 & 652.3 & 60.4 \\
\hline MEL-5 & 0.04 & 2.3 & 54.6 & 16.0 \\
\hline MEL-6 & 0.02 & 3.5 & 183.7 & 18.0 \\
\hline MEL-7 & 0.07 & 22.6 & 387.4 & 42.5 \\
\hline MEL-8 & 0.06 & 1.8 & 19.5 & 16.6 \\
\hline MEL-9 & 0.05 & 8.2 & 172.2 & 29.9 \\
\hline MEL-10 & 0.02 & 0.8 & 32.0 & $<2.0$ \\
\hline MEL-11 & 0.13 & 43.5 & 702.7 & 98.0 \\
\hline MEL-12 & 0.12 & 27.3 & 743.7 & 42.7 \\
\hline
\end{tabular}
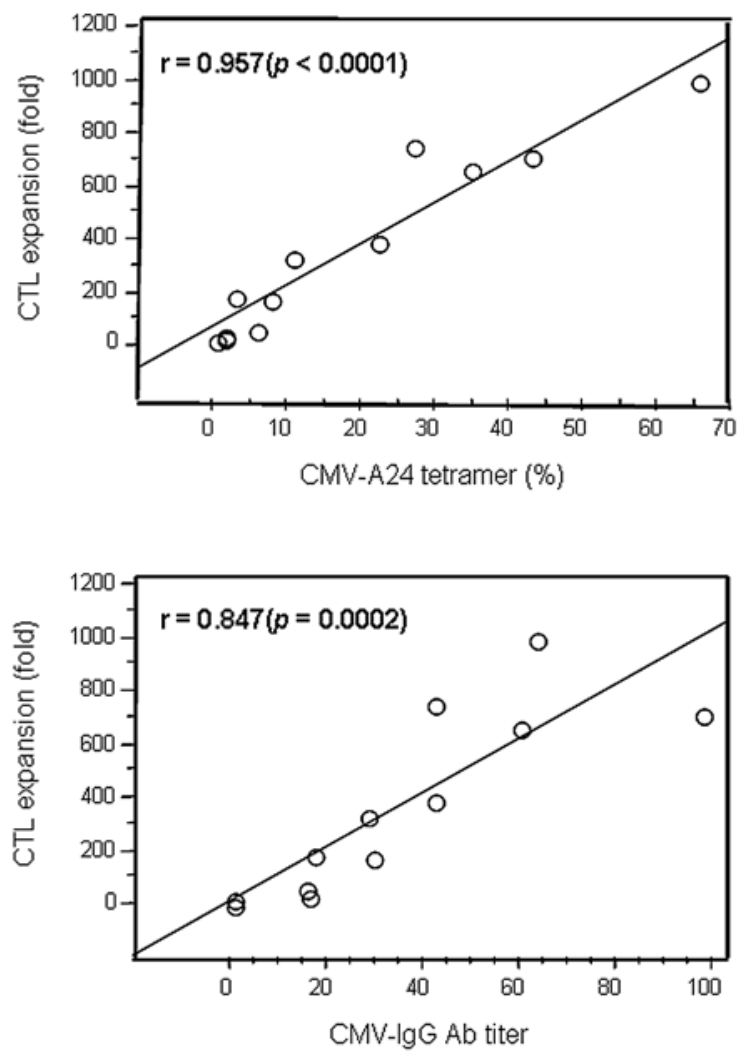

Figure 1. Correlation of CMV-specific CTL expansion efficiency with the frequency of tetramer ${ }^{+}$CTLs after expansion and anti-CMV IgG Ab titers. The correlation coefficient (r), was calculated and statistical difference was evaluated using a correlation analysis. The efficiency of CMV-specific CTL expansion was positively correlated with two parameters ( $\mathrm{r}=0.957$ vs. CMVA24 tetramer frequency; $r=0.847$ vs. anti-CMV IgG Ab titer).

efficiency to immunological parameters such as CMV A24 peptide-specific tetramer ${ }^{+}$CTL frequency and anti-CMV IgG antibody titer. Fig. 1 demonstrates that CTL expansion efficiency was closely related to both post-stimulation CMV 


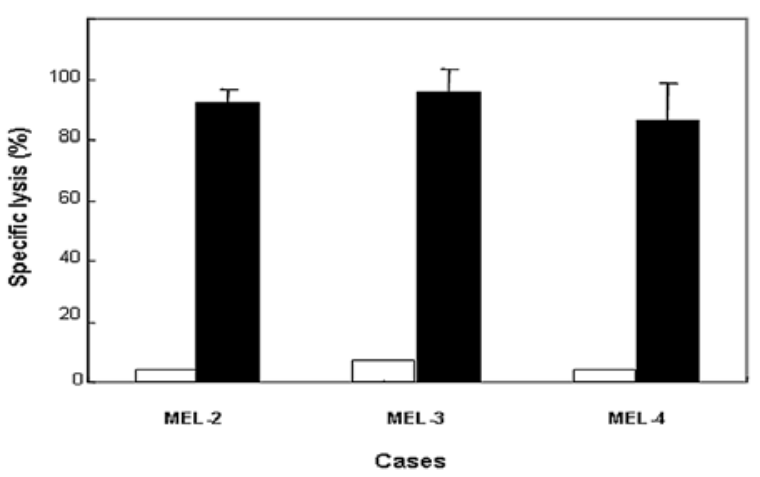

Figure 2. CTL killing activity of CMV A24 peptide-specific CTL lines derived from cases MEL-2, MEL-3 and MEL-4. TISI cells non-pulsed or pulsed with CMV A24 peptide were used as target cells. TISI cells were labeled with a fluorescence enhancing ligand, BATDA, and mixed with effector cells at an E/T ratio of 10 for $4 \mathrm{~h}$. Each column shows the the mean \pm SD for triplicate samples.
A24 tetramer ${ }^{+}$CTL frequency $(r=0.957)$ and anti-CMV IgG titer $(r=0.847)$.

CTL killing activity of expanded CMVpp65 A24 peptidespecific CTLs. Cultured CTLs from 3 melanoma cases showed strong killing activity against CMVpp65 A24 peptide-pulsed TISI cells (Fig. 2). In contrast, no significant killing activity was seen in irrelevant HIV A24 peptide-pulsed TISI cells.

TCR repertoire profiling in expanded CTL lines by specific anti-TCR MoAb. The TCR repertoire analysis using the TCR $\mathrm{V} ß$ repertoire kit revealed that CMVpp65-specific CTL lines from 3 melanoma cases showed a major anti-specific TCR MoAb-positive population which accounted for $>50 \%$ of all cells. Fig. 3 indicates staining profiles of CMVpp65-specific CTL lines from 3 melanoma cases using PE-labeled CMVpp65 A24 tetramer and FITC-anti-specific TCR MoAb (TRBV4
(A)

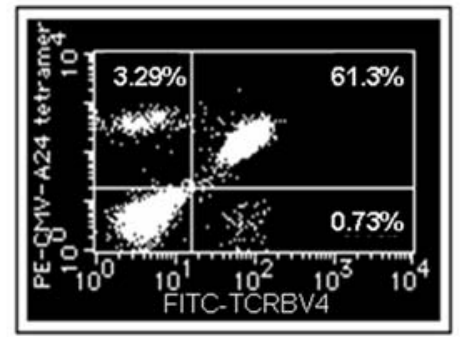

(B)

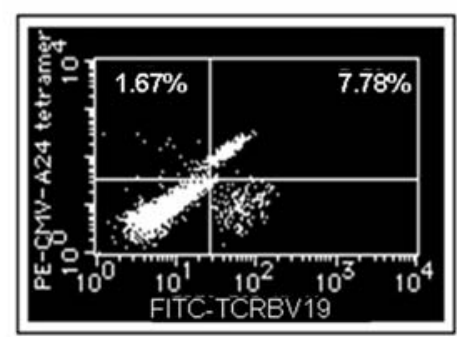

(C)

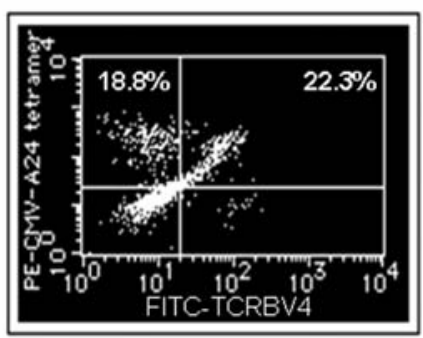

Figure 3. TCR repertoire profile of CMV A24-tetramer ${ }^{+}$CTLs from melanoma patients. Each panel shows CMV-specific CTLs positively stained with both CMV-A24 tetramer and specific anti-TCR MoAb. (A) Case MEL-2, (B) case MEL-3 and (C) case MEL-4.

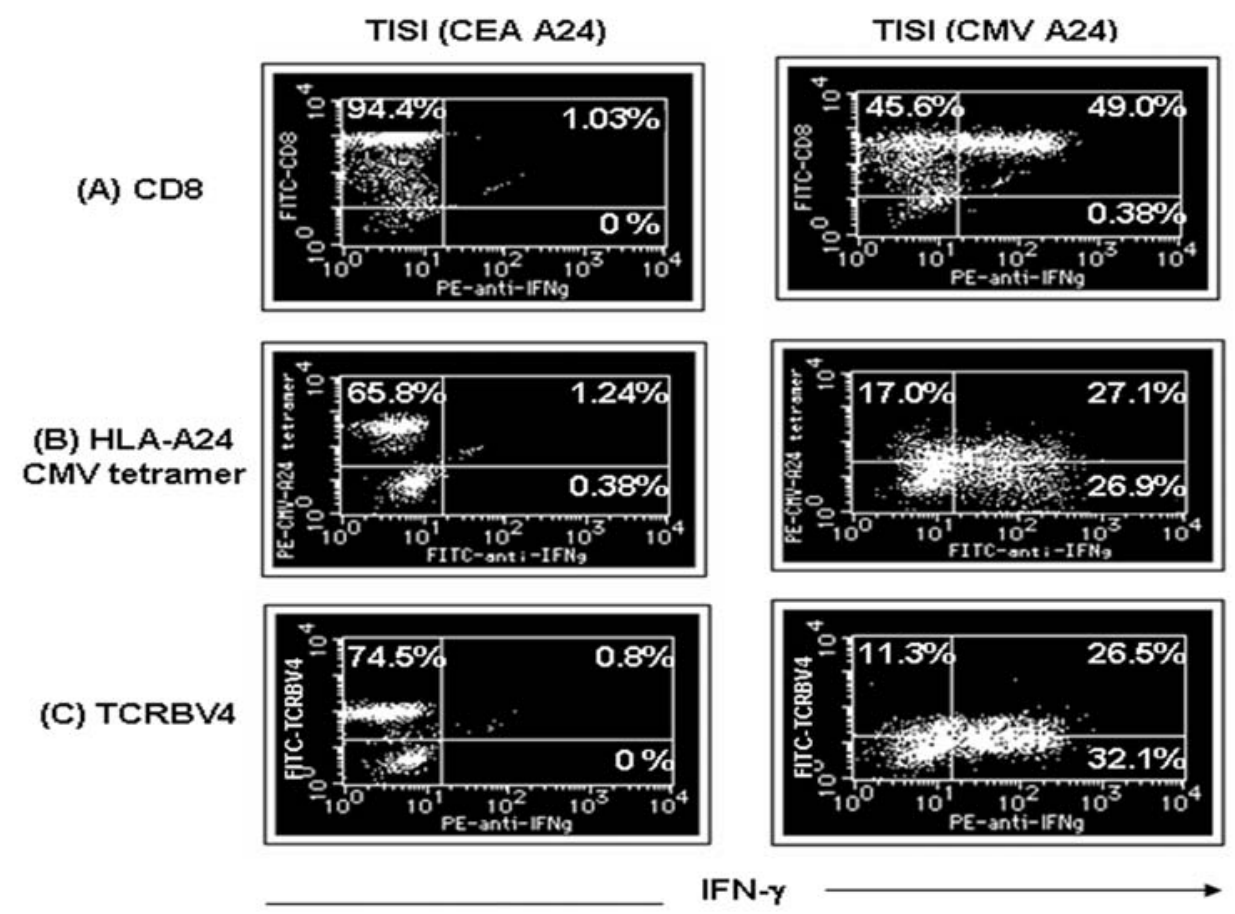

Figure 4. The relation of TCR repertoire profile in a CMV A24 peptide-specific CTL line from MEL-2 with IFN- $\gamma$ production. Left and right panels show IFN- $\gamma$ production from the CMV-specific CTL line stimulated with irrelevant or CMV A24 peptide-pulsed TISI cells, respectively. Stimulated CTLs were stained with anti-IFN- $\gamma$ Ab intracellularly and (A) anti-CD8, (B) HLA-A24 CMV tetramer or (C) anti-TCRBV4 MoAb. 


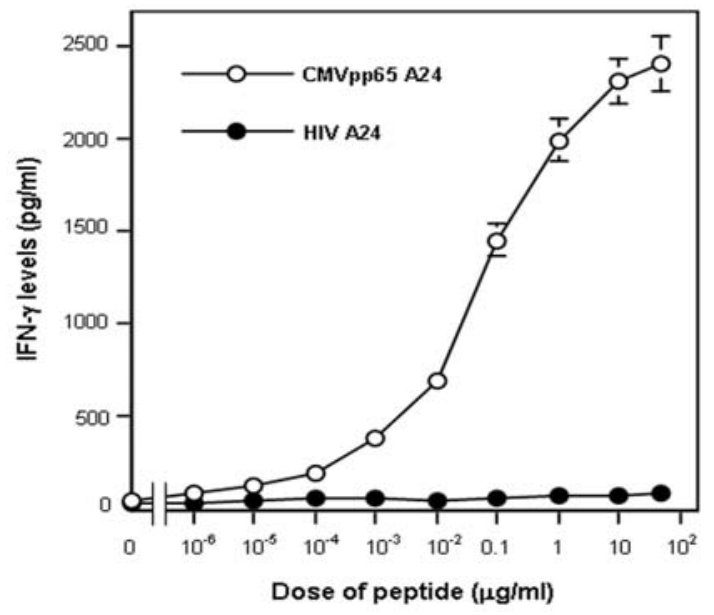

Firgure 5. IFN- $\gamma$ production from a CMV A24 peptide-specific CTL line sorted by anti-TCR MoAb. The CTL line from case MEL-2 was sorted by anti-TCRBV4 MoAb and stimulated with peptide-pulsed TISI cells, -o-; TISI cells pulsed with CMVpp65 A24 peptide, -•-; TISI cells pulsed with HIV A24 peptide.

for MEL-2 and MEL-4, and TRVB19 for MEL-3). CMVpp65 A24 tetramer ${ }^{+} /$anti-TCR $\mathrm{MoAb}^{+}$cells made up $94.9 \%$ (MEL-2), 82.3\% (MEL-3) and 54.2\% (MEL-4) of all CMVpp65 tetramer+ cells, respectively.

The relation of TCR repertoire profile to IFN- $\gamma$ production. Fig. 4 shows the correlation between TCR repertoire profile in CMVpp65-specific CTL from MEL-2 case and IFN- $\gamma$ production on CMVpp65 A24 peptide stimulation. Most CMVpp65 A24 tetramer ${ }^{+}$CTLs from MEL-2 were found to be TCRBV4-positive in Fig. 3. Approximately $70 \%$ of TCRVB4+ CTL and $62 \%$ of CMVpp65 A24 tetramer ${ }^{+}$ fractions were positive for IFN- $\gamma$ production on stimulation with CMVpp65 A24 peptide ${ }^{+}$target cells. In contrast, there was no significant IFN- $\gamma$ production on stimulation by irrelevant peptide-pulsed target cells. These results suggested that CMVpp65 A24 tetramer ${ }^{+} / \mathrm{TCRBV}^{+}$cells were the major populations responsible for peptide-specific IFN- $\gamma$ production. Finally, TCRBV4 ${ }^{+}$CTLs in cases MEL-2 and MEL-4 and TCRBV19+ CTLs in case MEL-3 were specifically sorted (purity $>95 \%$ ) using the autoMACS system and utilized for TCR gene cloning.

TISI-stimulated IFN- $\gamma$ production from CMVpp65 A24 peptidespecific CTL line sorted by TCR-specific MoAb. The CTL line from case MEL-2 sorted by FITC-labeled anti-TCRBV4
MoAb showed CMVpp65 A24-peptide specific IFN- $\gamma$ production (Fig. 5). The CMVpp65 A24-peptide specific CTL line exhibited a very potent IFN- $\gamma$ response to peptidetreated TISI cells at a dose as low as $100 \mathrm{pg} / \mathrm{ml}$. In contrast, there was no significant response to HIV A24 peptide-treated TISI cells.

TCR cDNA sequences in CMVpp65 A24 peptide-specific CTLs. Cloned TCR DNA-derived complementaritydetermining region (CDR)3 sequences from cases MEL-2, MEL-3 and MEL-4 are shown in Table II. The TCR repertoire used was TCRBV4-1*01 from MEL-2 and MEL-4, and TCRBV19*01 from case MEL-3, respectively.

TCR gene transduction into primary naive T cells in melanoma cases. The GFP gene transduction experiment after anti-CD3 and CD28 antibody-mediated $\mathrm{T}$ cell stimulation showed an efficient transduction $\left(\mathrm{CD}^{+} / \mathrm{GFP}^{+} \mathrm{T}\right.$ cell $54 \%$ out of all $\mathrm{CD}^{+} \mathrm{T}$ cells, data not shown). With $4 \mu \mathrm{g}$ of the TCR cDNA from case MEL-2 specific for CMVpp65 A24 peptide, the frequency of TCR-positive T Cells was 38.3\% (data not shown).

IFN- $\gamma$ production by TCR gene-transduced naive T cells on antigen stimulation. PBMCs from case MEL-2 were transduced with $4 \mu \mathrm{g}$ of TCR DNA specific for CMVpp65 A24 peptide by electroporation and used for co-culture with CMVpp65 peptide-pulsed TISI cells. The transduced PMBCs showed specific IFN- $\gamma$ production against CMVpp65 peptidepulsed TISI cells, but not CEA peptide-pulsed cells (Fig. 6). In contrast, PBMCs transduced with GFP cDNA did not respond.

\section{Discussion}

The development or re-activation of CMV infections in immunocompromised recipients of transplantations and highly advanced cancer patients who received a high dose of chemotherapy or irradiation remains difficult to stop. Recently, adoptive immunotherapy based on ex vivo CTL expansion and the transfer of effector cells against CMV has been focused on as a novel therapeutical tool, and evidence proving its worth is accumulating $(1-3,5,6)$.

Because of technical improvements in tetramer staining, several HLA class I-restricted epitope-derived tetramerpositive CTLs have been successfully detected and monitored in the clinical field. To date, many CTL epitopes derived from CMV pp65 protein have been confirmed for selected

Table II. CMVpp65 A24 peptide-specific TCR repertoire usage in 3 melanoma cases.

\begin{tabular}{llllll}
\hline Cases & TRBV & TRBD & TRBJ & TRBC & CDR3 sequence \\
\hline MEL-002 & TRBV4-1*01 & TRBD1 ${ }^{*} 01$ & TRBJ1-2*01 & TRBC1*01 & CASSEGQTQSKYGYTF \\
MEL-003 & TRBV19*01 & TRBD1 ${ }^{*} 01$ & TRBJ1-2*01 & TRBC1*01 & CASSPQTSSHGYTF \\
MEL-004 & TRBV4-1 ${ }^{*} 01$ & TRBD2*01 & TRBJ2-1*01 & TRBC2 & CASSPTAGSSYNEQFF \\
\hline
\end{tabular}

Cloned TCR DNA-derived complementarity-determining region (CDR)3 sequences from cases MEL-2, MEL-3 and MEL-4 are shown. Segments V, D, J and C were identified using a tool at the IMTG web site (JunctionAnalysis, http://imgt.cines.fr/). 


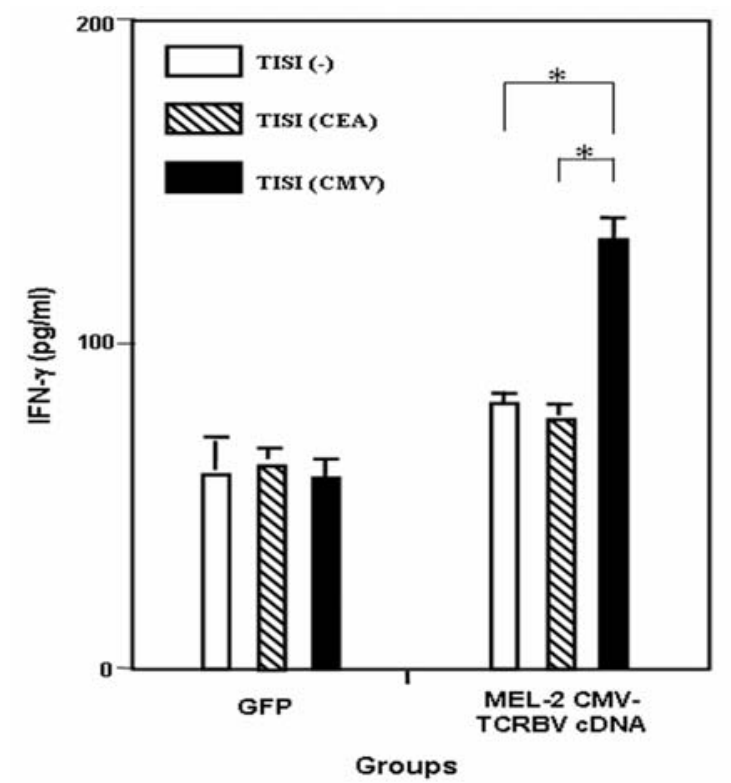

Figure 6. IFN- $\gamma$ production from CMV A24 peptide-specific TCR genetransduced naive $\mathrm{T}$ cells on antigen stimulation. PBMCs from case MEL-2 were transduced with CMV A24 peptide-specific TCRBV cDNA by electroporation and used for co-culture with peptide-pulsed TISI cells. White bars, non-pulsed TISI cells; striped bars, CEA A24 peptide-pulsed TISI cells; black bars, CMVpp65 A24 peptide-pulsed TISI cells. ${ }^{*} \mathrm{P}<0.05$.

HLA class I alleles such as HLA-A*0101, A*0201, A*1101, $\mathrm{A}^{*} 2402, \mathrm{~A} 33, \mathrm{~B}^{*} 0702$ and $\mathrm{B}^{*} 3501$ (7-9). Interestingly, some researchers reported HLA-CW-specific epitopes, another HLA-A*2402-restricted epitope (VYALPLKML) and proposed HLA-A24 supertype (A*2301, A*2402 and A*3001) CTL epitope (10-12). In particular, HLA-A*0201-CMVpp65 peptide (NLVPMVATV) tetramer has been frequently used for CTL monitoring and functional evaluations in allogenic hematopoietic stem cell transplantation (SCT) and HIV-infected patients (13-16). Ozdemir et al demonstrated that frequencies of CMV-specific CD8 ${ }^{+} \mathrm{T}$ cells were significantly higher in those subjects who experienced early antigenemia relative to those who did not (2.2 vs $0.75 \%)$ (17). Meanwhile, HLAA*2402-CMVpp65 peptide (QYDPVAALF) tetramer was also shown to be effective for the early detection and specific and sensitive monitoring of blood $\mathrm{CMV}$-specific $\mathrm{CD} 8^{+} \mathrm{T}$ cells in allogeneic SCT patients $(18,19)$. More importantly, Gondo et al demonstrated that $\mathrm{CMV}$-specific $\mathrm{CD} 8^{+} \mathrm{T}$ cells were constructed earlier in allogeneic peripheral blood SCT patients than allogeneic bone marrow SCT patients (18).

In order to apply CMV-specific adoptive CTLs to the clinical field, sufficient quantities of CTLs with efficient killing activity will be needed. In vitro expansion methods to generate a substantial number of HLA-A*0201-positive CTLs using autologous DCs, lymphoblastoid cell lines (LCLs) and anti-CD3 antibody have been demonstrated $(5,20,21)$. Specifically, Carlsson et al reported that in vitro stimulation with adenovirus-mediated CMVpp65 gene-transduced DCs and peptide-pulsed monocytes enabled HLA-A 0201 tetramer ${ }^{+}$CMV-specific CTLs to expand up to 1000 -fold in 2 weeks (22). Meanwhile, Provenzano et al demonstrated that HLA-A*2402 tetramer ${ }^{+}$CTLs could be selected and expanded from CMV-seropositive donors bearing HLA-
A*2402 and HLA-A*0101 (23). In the present study, we tried to characterize and expand HLA-A*2402 CMVpp65 tetramer ${ }^{+}$ CTLs from HLA-24-positive metastatic melanoma patients, and eventually demonstrated that CTL expansion efficiency was closely related to both post-stimulation CMV tetramer ${ }^{+}$ CTL frequency and anti-CMV IgG titer. This is a novel finding regarding in vitro CMV-HLA-A24 epitope specific CTL expansion based on metastatic cancer patient-derived PBLs.

A previous study using healthy volunteer PBLs found a good response of CMV HLA-A24 tetramer+ CTLs after in vitro stimulation with autologoud DCs and potent killing (4). The current results using metastatic melanoma PBLs showed much higher frequencies of CMV HLA-A24 tetramer+ CTL and expansion efficiency than in healthy volunteers. Additionally, the frequency and antibody titers were also greater for CMVseropositive melanoma patients than volunteers. Probable reasons for this are as follows: i) melanoma patients are much older than volunteers and have high anti-CMV antibody titers, and ii) CTL killing activity was not impaired in the melanoma patients shown in Fig. 6, in contrast to a report that CMV-specific CTLs in immunosuppressive patients showed a decreased functional response to CMV-peptide (24). Our previous report (25) that autologous DC-mediated stimulated CTLs against HLA-A24 melanoma peptide cocktail showed less potent killing activity than healthy volunteers might demonstrate the greater immunogenicity of CMVspecific CTL epitopes.

Finally, we were successful in cloning CMVpp65 HLAA24 peptide-specific TCR cDNA from in vitro expanded CTL lines derived from melanoma patients. Additionally, CMVpp65 HLA-A24 peptide-specific TCR cDNA was transduced into naive $\mathrm{T}$ cells from the patients and functionally reconstructed. Hamel et al (26) also cloned a CMV-HLA-A2 peptide-specific TCR gene from in vitro stimulated CTLs using autologous DCs infected with a CMVpp65 genecontaining adenoviral-vector. However, the CMV-specific CTL expansion efficiency was around 200-fold, lower than our expansion data. These results suggest the in vitro expansion method described here and TCR cDNA cloning technology to be good tools for the clinical application of expanded CMV-specific CTLs in immunocompromised patients.

\section{Acknowledgements}

We thank Dr Tohru Mochizuki, Shizuoka Cancer Center Research Institute, for supplying several synthetic peptides. This study was supported in part by a grant from the cooperation of Innovative Technology and Advanced Research in the Evolutional Area (CITY AREA) program from the Ministry of Education, Culture, Sports, Science and Technology.

\section{References}

1. Riddell SR, Watanabe KS, Goodrich JM, Li CR, Agha ME and Greenberg PD: Restoration of viral immunity in immunodeficient humans by the adoptive transfer of $\mathrm{T}$ cell clones. Science 257 : 238-241, 1992.

2. Walter EA, Greenberg PD, Gilbert MJ, Finch RJ, Watanabe KS, Thomas ED and Riddell SR: Reconstitution of cellular immunity against cytomegalovirus in recipients of allogeneic bone marrow by transfer of T-cell clones from the donor. N Engl J Med 333: 1038-1044, 1995. 
3. Lucas KG, Sun Q, Burton RL, et al: A phase I-II trial to examine the toxicity of CMV- and EBV-specific cytotoxic T lymphocytes when used for prophylaxis against EBV and CMV disease in recipients of CD34-selected/T cell-depleted stem cell transplants. Hum Gene Ther 11: 1453-1463, 2000.

4. Akiyama Y, Maruyama K, Mochizuki, Sasaki K, Takaue Y and Yamaguchi K: Identification of HLA-A24-restricted CTL epitope encoded by the matrix protein pp65 of human cytomegalovirus. Immunol Lett 83: 21-30, 2002.

5. Einsele H, Rauser G, Grigoleit U, Hebart H, Sinzger C, Riegler S and Jahn G: Induction of CMV-specific T-cell lines using Agpresenting cells pulsed with CMV protein or peptide. Cytotherapy 4: 49-54, 2002

6. Hensel N, Melenhorst JJ, Bradstock K, Schwarer AP, Eniafe R, Nakamura $\mathrm{R}$ and Barrett AJ: Flow cytometric quantitation and characterization of the T-lymphocyte memory response to CMV in healthy donors. Cytotherapy 4: 29-40, 2002.

7. Forman SJ and Zaia JA: Treatment and prevention of cytomegalovirus pneumonia after bone marrow transplantation: where do we stand? Blood 83: 2392-2398, 1994.

8. Gallant JE, Moore RD, Richman DD, Keruly J and Chaisson RE: Incidence and natural history of cytomegalovirus disease in patients with advanced human immunodeficiency virus disease treated with zidovudine. The Zidovudine Epidemiology Study Group. J Infect Dis 166: 1223-1227, 1992.

9. Kuzushima K, Hayashi N, Kimura H and Tsurumi T: Efficient identification of HLA-A ${ }^{*} 2402$-restricted cytomegalovirus-specific $\mathrm{CD}^{+} \mathrm{T}$-cell epitopes by a computer algorithm and an enzymelinked immunospot assay. Blood 98: 1872-1881, 2001.

10. Masuoka M, Yoshimuta T, Hamada M, et al: Identification of the HLA-A24 peptide epitope within cytomegalovirus protein pp65 recognized by CMV-specific cytotoxic T lymphocytes. Viral Immunol 14: 369-377, 2001.

11. Kondo E, Akatsuka Y, Kuzushima K, et al: Identification of novel CTL epitopes of CMV-pp65 presented by a variety of HLA alleles. Blood 103: 630-638, 2004.

12. Burrows SR, Elkington RA, Miles IJ, et al: Promiscuous CTL recognition of viral epitopes on multiple human leukocyte antigens: biological validation of the proposed HLA A24 supertype. J Immunol 171: 1407-1412, 2003.

13. Lim JB, Provenzano M, Kwon OH, Bettinotti M, Caruccio L, Nagorsen D and Stroncek D: Identification of HLA-A33-restricted CMV pp65 epitopes as common targets for CD8(+) CMVspecific cytotoxic T lymphocytes. Exp Hematol 34: 296-307, 2006.

14. Engstrand M, Tournay C, Peyrat MA, et al: Characterization of CMVpp65-specific $\mathrm{CD} 8^{+} \mathrm{T}$ lymphocytes using MHC tetramers in kidney transplant patients and healthy participants. Trasplantation 69: 2243-2250, 2000.

15. Aubert G, Hassan-Walker AF, Madrigal JA, et al: Cytomegalovirus-specific cellular immune responses and viremia in receipients of allogeneic stem cell traansplants. J Infect Dis 184: 955-963, 2001
16. Grantama JW, van Esser JWJ, Lamers CHJ, et al: Tetramerbased quantification of cytomegalovirus (CMV)-specific $\mathrm{CD}^{+}$ $\mathrm{T}$ lymphocytes in T-cell-depleted stem cell grafts and after transplantation may identify patients at risk for progressive CMV infection. Blood 98: 1358-1364, 2001.

17. Ozdemir E, St. John LS, Gillespie G, Rowland-Jones S, Champlin RE, Molldrem JJ and Komanduri KV: Cytomegalovirus reactivation following allogeneic stem cell transplantation is associated with the presence of dysfunctional antigen-specific CD8 ${ }^{+}$T cells. Blood 100: 3690-3697, 2002.

18. Gondo H, Himeji D, Kamezaki K, et al: Reconstitution of HLAA2402-restricted cytomegalovirus-specific T-cells following stem cell transplantation. Int J Hematol 80: 441-448, 2004.

19. Morita Y, Hosokawa M, Ebisawa M, Sugita T, Miura O, Takaue Y and Heike Y: Evaluation of cytomegalovirus-specific cytotoxic T-lymphocytes in patients with the HLA-A*02 or HLA-A* 24 phenotype undergoing hematopoietic stem cell transplantation. Bone Marrow Transplant 36: 803-811, 2005.

20. Szmania S, Galloway A, Bruorton M, et al: Isolation and expansion of cytomegalovirus-specific cytotoxic $\mathrm{T}$ lymphocytes to clinical scale from a single blood draw using dendritic cells and HLA-tetramers. Blood 98: 505-512, 2001.

21. Watanabe N, Kamachi Y, Koyama N, et al: Expansion of human CMV-specific cytotoxic T lymphocytes to a clinical scale: a simple culture system using tetrameric HLA-peptide complexes. Cytotherapy 6: 514-522, 2004.

22. Carlsson B, Cheng WS, Totterman TH and Essand M: Ex vivo stimulation of cytomegalovirus (CMV)-specific $\mathrm{T}$ cells using CMV pp65-modified dendritic cells as stimulators. Br J Hematol 121: 428-438, 2003.

23. Provenzano M, Lim JB, Mocellin S, Monsurro V, Bettinotti M, Marincola FM and Stroncek D: The matrix protein pp65341-350: a peptide that induces ex vivo stimulation and in vitro expansion of CMV-specific CD8 ${ }^{+} \mathrm{T}$ cells in subjects bearing either HLAA*2402 or A*0101 allele. Transfusion 43: 1567-1574, 2003.

24. Engstrand M, Lidehall AK, Totterman TH, Herrman B, Eriksson BM and Korsgren O: Cellular responses to cytomegalovirus in immunosuppressed patients: circulating $\mathrm{CD}^{+} \mathrm{T}$ cells recognizing CMVpp65 are present but display functional impairment. Clin Exp Immunol 132: 96-104, 2003.

25. Akiyama Y, Maruyama K, Nara N, et al: Cytotoxic $\mathrm{T}$ cell induction against human malignant melanoma cells using HLAA24-restricted melanoma peptide cocktail. Anticancer Res 24: 571-578, 2004.

26. Hamel Y, Rohrlich P, Baron V, et al: Characterization of antigenspecific repertoire diversity following in vitro restimulation by a recombinant adenovirus expressing human cytomegalovirus pp65. Eur J Immunol 33: 760-768, 2003. 